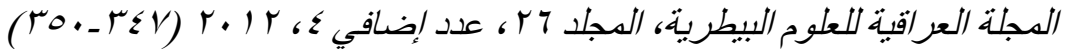

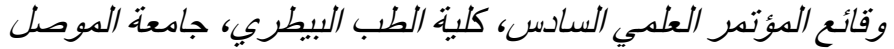

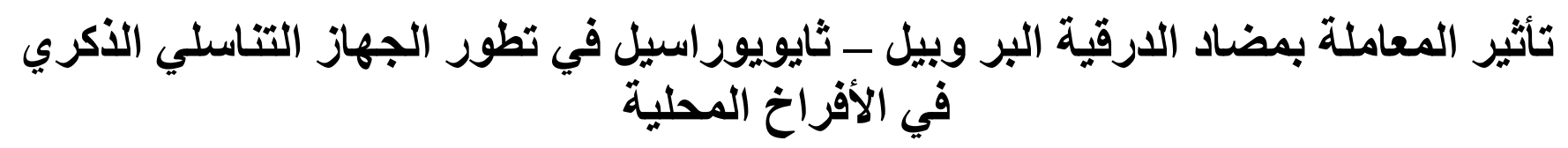

\author{
إنعام عناد جبوري و ناظم أحمد حسن القاسم \\ فرع الفسلجة والكيمياء الحياتية والأدوية، كلية الطب البيطري، جامعة الموصل، الموصل، العراق \\ Email:nadhem_1957@yahoo.com
}

الخلاصة

تهدف الدراسة الحالية الى معرفة تاثيرمضاد الدرفية (البر وبيل ثايويور اسيل) في تطور الجهاز التناسلي الذكري للأفر اخخ المحلية.

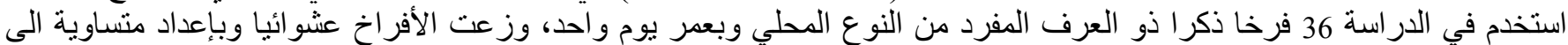

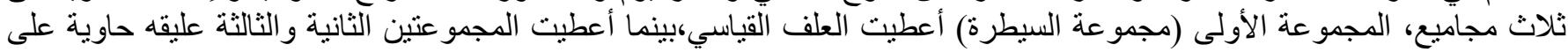

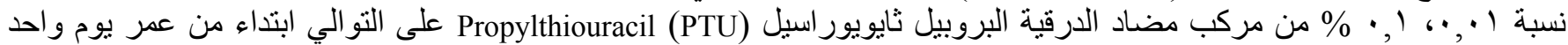

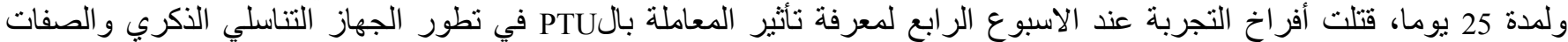

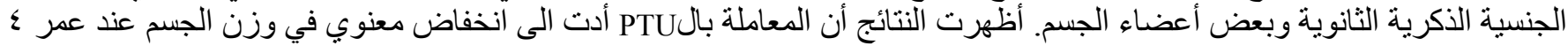

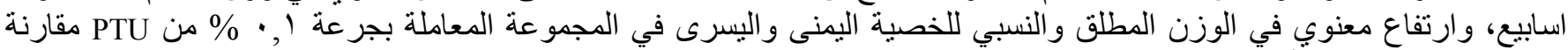

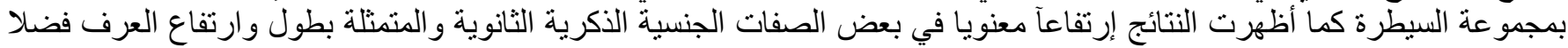

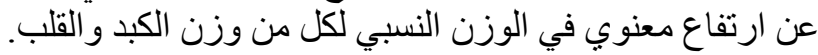

\title{
Effect of antithyroid propyl thiouracil on male reproductive system development in local chicks
}

\section{N.A.H. Alkasim and I.E. Jobury}

Department of Physiology, Biochemistry \& Pharmacology, College of Veterinary Medicine, University of Mosul, Mosul, Iraq

\begin{abstract}
The aim of this experiment is to study the effects of antithyroid agent (Propylthiouracil, PTU) on domestic chicks male reproductive system. 36 local male one day old chicks were used. Chicks were distributed randomly to three groups, first group (control group) fed standard diet, while 2 nd and 3 rd groups were fed diet contain 0.01 and $0.1 \%$ of antithyroid agent (Propylthiouracil PTU) respectively, from 1 - 25 days of age. Chicks were killed at the end of 4th week in order to study the effects of PTU treatment on some male reproductive system and secondary sexual characteristic as well as some body organs. Results revealed that PTU treatment significantly decreased body weight, significantly increased right and left testis percentage and absolute weights in group treated with $0.1 \%$ PTU compared with the control group. Treatment also significantly increase comb length and height as well as in liver and heart weights.
\end{abstract}

Available online at http://www.vetmedmosul.org/ijvs

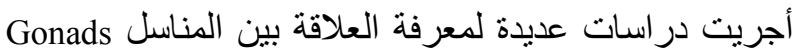

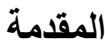

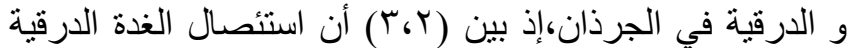

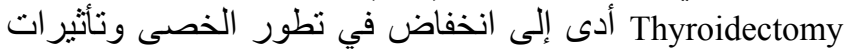
سلبية في نمو وتطور النطف مع التقليل من الرغورف النبة الجنسية لللذكور.

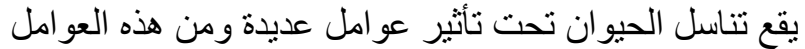

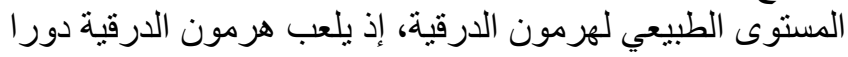

مهما في تطور الخصى في الجرذان (1). 


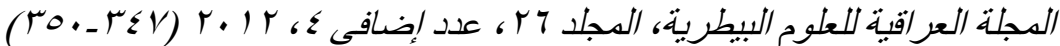

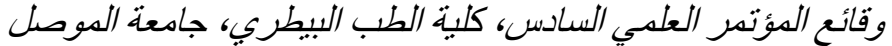

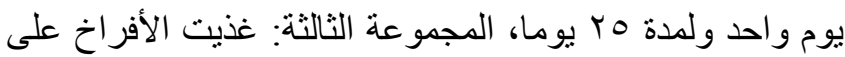

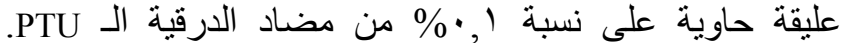

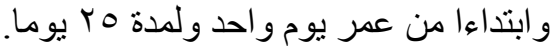

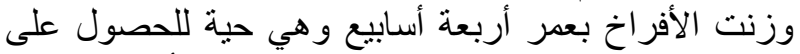

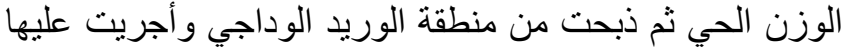

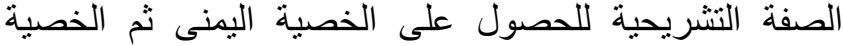

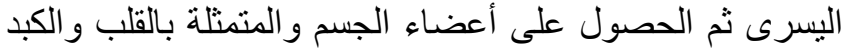

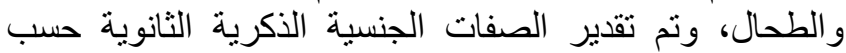
الطريقة التي وصفها (10) (10).

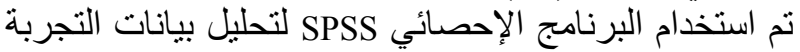

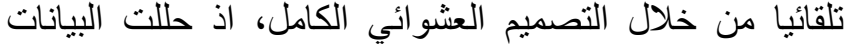

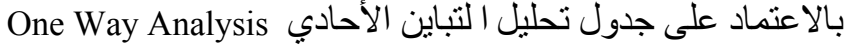
of Variance Standard Error المعنوية بين مجاميع التجربة وعند مستوى معنوية 0.05 >

النتائجج

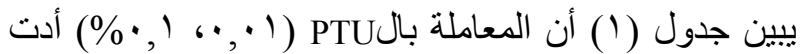

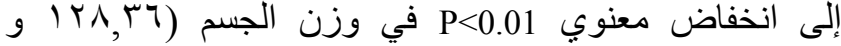

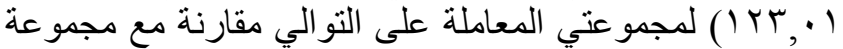

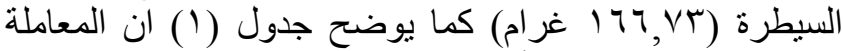

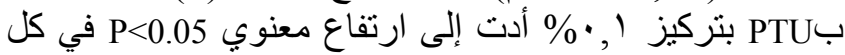
من الوزن المطلق والوزن النسبي للخصية اليمنى واليسرى الئرى

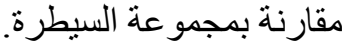

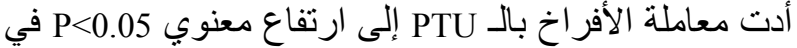

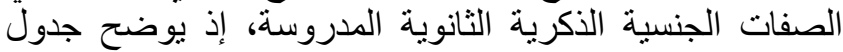

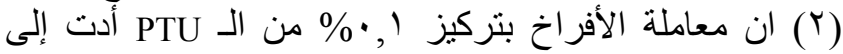

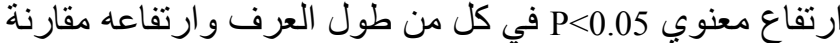

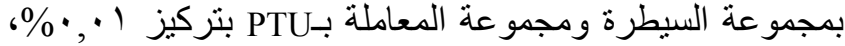

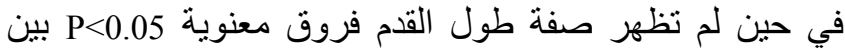
مجاميع الدراسة الثلاثة (مجموعة السيطرة ومجموعت معتي المعاملة

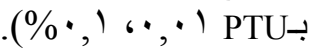

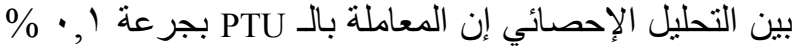

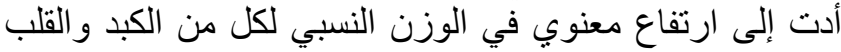

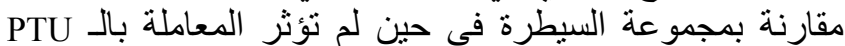

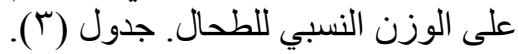

\section{المناقشة}

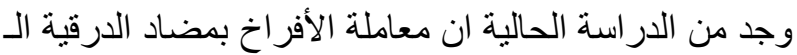
PTU

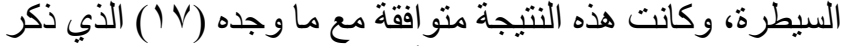

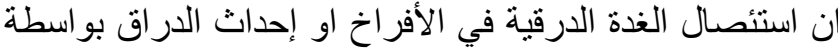

فيما بين (ع) إن لهرمون الدرقية دورا في فترة النمو الحرجة

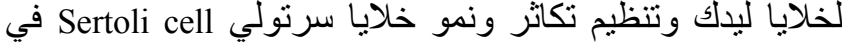

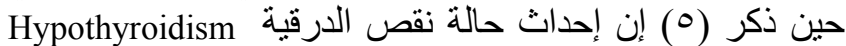

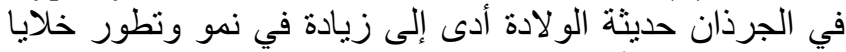

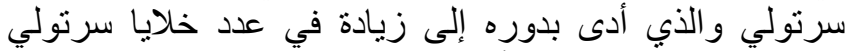

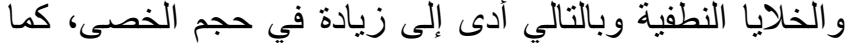

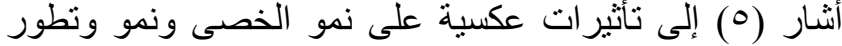

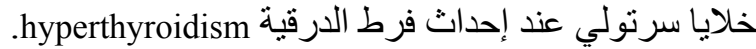

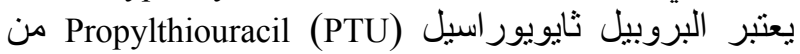

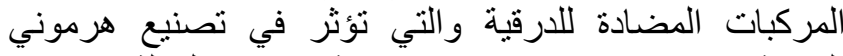
الدرقية thyroid hormones (T) كما يؤثر في تحويل الثئن الثايروكسين $T_{3}$ thyroxine $\left(T_{4}\right)$ triiodo thyronine

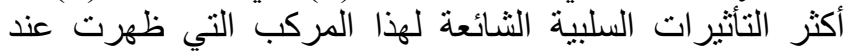

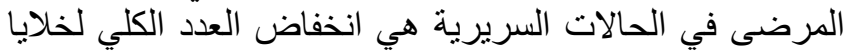

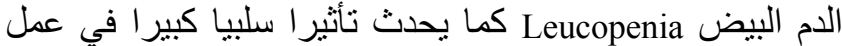

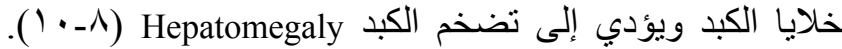
وبين (9) أن ليس لهذا المركب تأثبر ات واضئ واضحة في خلايا خصى

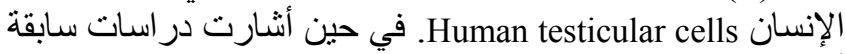

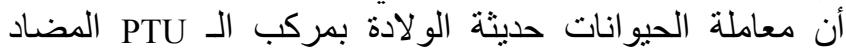

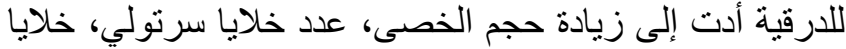
ليدك وزيادة في إنتاج النطف اليومية في الجرذان والئان الفئران

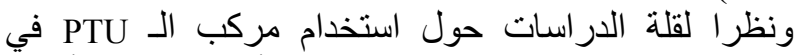
الطيور هدفت الدراسة الحالية إلى معرفة تأثير معاملة الأفراخ

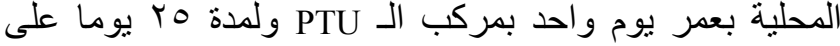
تطور الجهاز التناسلي الذكري و الصفات الجنسية الذكرية الثانوية

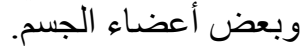

\section{المواد وطر ائق العمل}

استخدم في التجربة جب فرخا ذكرا ذو العرف المفرد من

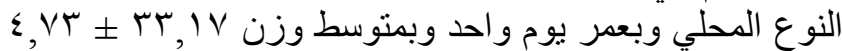

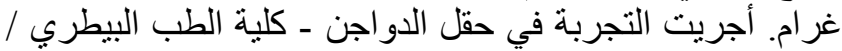
جامعة الموصل. إذ تم إعداد القاعة بغسلها جيدا ثما ثم تعقيمها

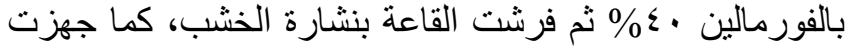

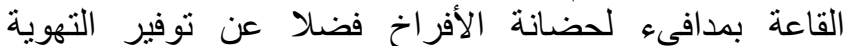
والإضاءة المناسبة للأفر اخ. غذيت الأيت الأفر اخ على العليقة القياسية

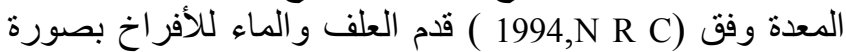

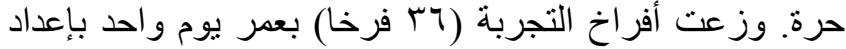

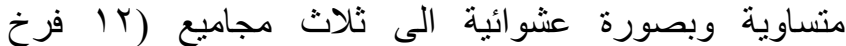

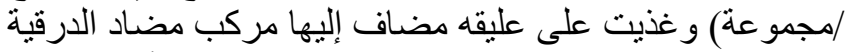

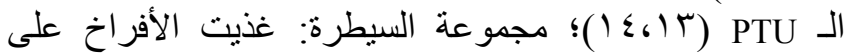

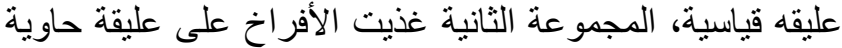

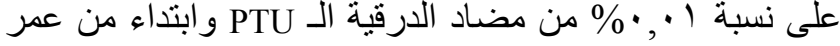




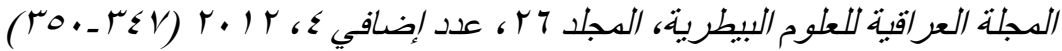

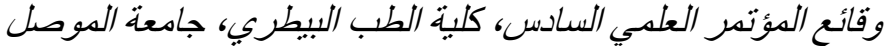

انخفاض معنوي في تعبير الحامض النووي الرايبوزي الرسول

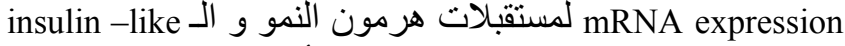
growth factor (IGF-1) الدرقية يؤدي الى انخفاض في معدل النمو.
الـ goiterogen في الأفر اخ عن طريق التغذية أدت إلى انخفاض

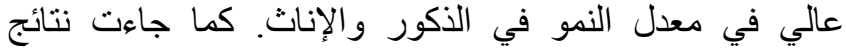

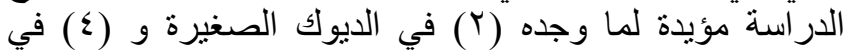

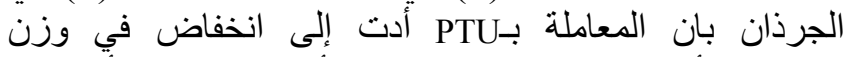

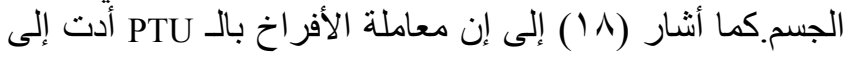

جدول (1): تأثير معاملة ذكور الأفر اخ بالآPTU على وزن الجسم والوزن المطلق و النسبي للخصية اليمنى واليسرى عند الاسبوع الرابع من عمر الأفراخ. تناكير

\begin{tabular}{|c|c|c|c|c|c|}
\hline \multicolumn{5}{|c|}{ الصفات المدروسة } & \multirow[b]{2}{*}{ المجاميع } \\
\hline 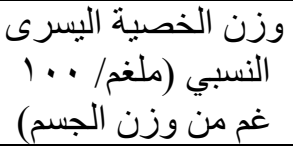 & 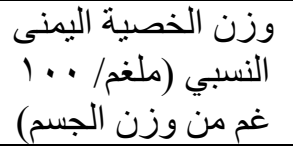 & وزن الخصى المطلة & وزن الينى المطلقة & وزن الجسم & \\
\hline $\begin{array}{c}3.43 \pm 21.70 \\
\mathrm{a}\end{array}$ & $\begin{array}{c}2.21 \pm 13.71 \\
\mathrm{a}\end{array}$ & $\begin{array}{c}2.72 \pm 23.65 \\
\mathrm{a}\end{array}$ & $\begin{array}{c}5.09 \pm 21.67 \\
\mathrm{a}\end{array}$ & $\begin{array}{c}9.59 \pm 166.73 \\
\mathrm{a}\end{array}$ & مجمو عة السيطرة \\
\hline $\begin{array}{c}4.59 \pm 18.50 \\
\mathrm{a}\end{array}$ & $\begin{array}{c}4.93 \pm 16.75 \\
\mathrm{ab}\end{array}$ & $\begin{array}{c}5.73 \pm 24.00 \\
\mathrm{a}\end{array}$ & $\begin{array}{c}18.31 \pm 23.45 \\
\mathrm{ab}\end{array}$ & $\begin{array}{c}8.39 \pm 128.36 \\
\mathrm{~b}\end{array}$ & 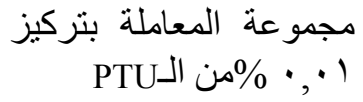 \\
\hline $\begin{array}{c}7.83 \pm 37.67 \\
b\end{array}$ & $\begin{array}{c}5.52 \pm 28.03 \\
b\end{array}$ & $\begin{array}{c}8.45 \pm 40.60 \\
\mathrm{~b}\end{array}$ & $\begin{array}{c}22.37 \pm 9.68 \\
b\end{array}$ & $\begin{array}{c}5.58 \pm 123.01 \\
\mathrm{~b}\end{array}$ & 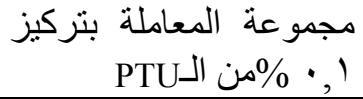 \\
\hline
\end{tabular}

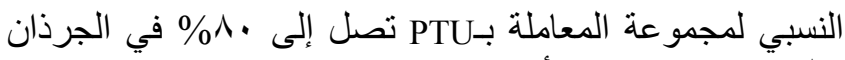
و الفئران و 97\% في الأفر اخ (Yr).

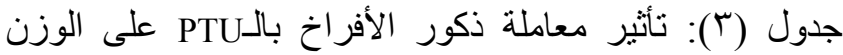

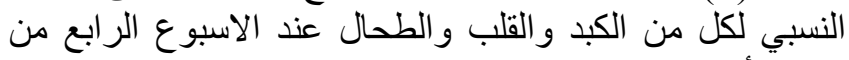
عمر ألأفر اخ.

\begin{tabular}{|c|c|c|c|}
\hline \multicolumn{3}{|c|}{ الوزن النسبي } & \multirow{5}{*}{ المجاميع } \\
\hline الطحال & القلب & الكبد & \\
\hline (ملغم/ & (ملغم/ & (ملغم/ & \\
\hline ل . 1 غم & ل . 1 غم & ... & \\
\hline الجسم) & من وزن & من وزن & \\
\hline \pm 185.97 & \pm 708.13 & \pm 2829.86 & مجمو عة السيطرة \\
\hline 28.50 & 30.74 & 206.74 & \\
\hline $\mathrm{a}$ & $\mathrm{a}$ & $\mathrm{a}$ & \\
\hline
\end{tabular}

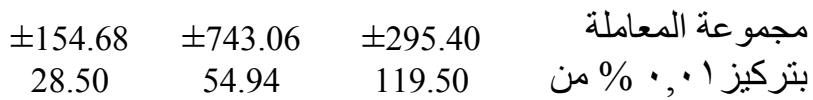
$\begin{array}{llll}\text { a } & \text { a } & \text { a } & \text { PTU } 150\end{array}$

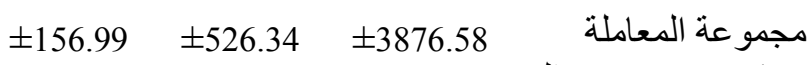

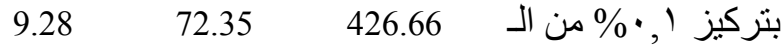
\begin{tabular}{llll}
$a$ & $b$ & $b$ & PTU \\
\hline
\end{tabular} الأحرف المختلفة في العمود الواحد تدل على وجود فروق معنوية

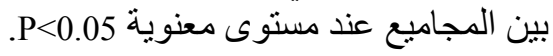

جدول (r): تأثير معاملة ذكور الأفراخ بال PTU على بعض

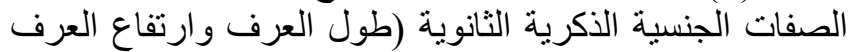
وطول القدم) عند الأسبوع الأرية البع من عمر الأفراخ.

\begin{tabular}{|c|c|c|c|}
\hline \multicolumn{3}{|c|}{ الصفات الجنسية الذكرية الثانوية } & \multirow[b]{2}{*}{ المجاميع } \\
\hline 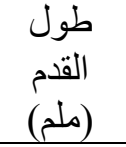 & $\begin{array}{l}\text { العرتفاع } \\
\text { العلم) }\end{array}$ & 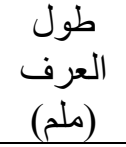 & \\
\hline \pm 35.43 & \pm 2.51 & \pm 13.17 & مجمو عة السيطرة \\
\hline 1.31 & 0.44 & 0.97 & \\
\hline $\mathrm{a}$ & $\mathrm{a}$ & $\mathrm{a}$ & \\
\hline \pm 37.20 & \pm 4.43 & \pm 14.70 & مجمو عة المعاملة \\
\hline 3.37 & 0.64 & 0.56 & بتركيز 1 •, • \% من \\
\hline $\mathrm{a}$ & $\mathrm{a}$ & $\mathrm{a}$ & الـ \\
\hline \pm 47.78 & \pm 7.13 & \pm 21.40 & مجمو عة المعاملة \\
\hline 13.90 & 0.90 & 0.64 & بتركيز ا, •\% من الـ \\
\hline $\mathrm{a}$ & $\mathrm{b}$ & $\mathrm{b}$ & PTU \\
\hline \multicolumn{4}{|c|}{ 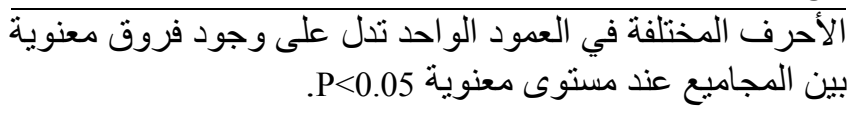 } \\
\hline
\end{tabular}




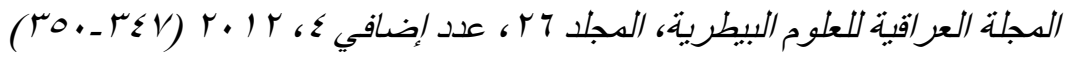

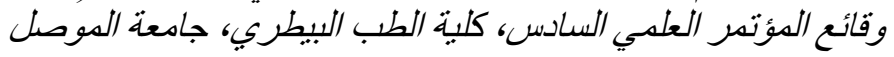

9. Deidiker R. Demllo DE. Propylthiouracil-induced fulminant hepatitis:case report and review of the literature.Pediatr. Pathol.Lab. Med. 1996; 16: 845-852.

10. Jonas MM. Eidson MS. Propylthiouracil hepatotoxicity:two pediatric cases and review of the literature. J. Pediatr.Gastroenterol.Nutr. 1988; 7: 776-779.

11. Hess RA. Cooke PS. Bunick D. Kirby JD. Adult testicular enlargement induced by neonatal hypothyroidism is accompanied by increased Sertoli and germ cell numbers. Endocrinology. 1993; 132: 2607-2613.

12. Joyce KL. Porcelli J. Cooke PS. Neonatal goitrogen treatment increases adult testis size and sperm production in the mouse.J.Androl. 1993 ; 1: 448-455.

13. Aklaghi A. Zamiri MJ. Effect of transient Prepubertal hypothyroidism on serm testosterone level and seminal characteristics of chickens. Iran. J. Vet. Res. 2007; 8(1):23-31.

14. John DK. Maithili VM. Debbie H. David LK. Effect of Transient Prepubertal 6-N-Propyl -2-Thiouracil Treatment on Testis Development and Function in the Domestic Fowl. 1996; 55:910-916.

1.اللهيبي، ياسين محمد عبد الله. تأثير جرعات مختلفة من الاسترادايول

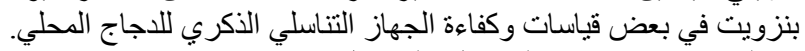

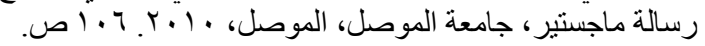

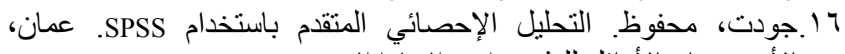

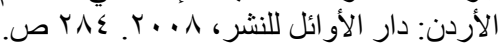

17. Pandey JN. Bajaj MR. Effect of thiouracil administration on growth rate and carcass yield of Australorp chicks.Haryana Agric.Univ.J.Res. $1974 ; 4: 153-157$.

18. Tsukada A. Ohkubo T. Sakaguchi K. Tanaka M. Nakashima K. Hayashida K. Wakita M. Hoshino S. Thyroid hormones are in volved in insulin-like growth factor (IGF)-1 production by regulating growth hormone receptor (GHR)in the chicken. Poult.Avian Biol.Rev. 1996 ; 8:253.

19. Cooke PS.Hess RA. Porcelli J. Meisami E. Increased sperm production in adult rats after transient neonatal hypothyroidism. Endocrinology. $1991 ; 129: 244-248$.

20. Cooke PS. Meisami E. Early hypothyroidism in rats causes increased adult testis and reproductive size but does not change testosterone level.Endocrinology. 1991;129:237-243.

21. Joyce KL.Porcelli J. Cooke PS. Neonatal goitrogen treatment increases adult testis size and sperm production in the mouse. J.Androl. 1993; 14:448-455.

22. Kirby JD. Mankar MV. Hardesty D. Kreider DL. Effects of transient prepubertal 6-N-propyl -2-thiouracil treatment on testis development and function in domestic fowl.Biol.Reprod. 1996; 55:910-916.

23. Knowlton JA. Siopes TD. Roads ML. Kirby JD. Effects of transient treatment with 6-N-propyl -2-thiouracil on testis development and function in breeder turkeys.poultry Sci. 1999 78:999-1005

24. Kirby JD. Jetton E. Cooke PS. Hess RA. Bunick D. Ackland JF. Turek Fw. Schwartz NB. Developmental hormonal profiles accompanying the neonatal hypothyroidism induced increases in adult testis size and sperm production in the rat. Endocrinology. $1992 ; 131: 559-565$.

25. Hermansoon H. Effects on the Reproductive system in Domestic Fowl (Gallus domesticus)after Embryonic Exposure to Estrogenic Substances. [Doctoral thesis] Swedish: Swedish University of Agricultural Sciences.2007. 115P.

26. Balthazat J. Hendrick JC. Steroidal control of plasma lutenizing hormone, comb growth and sexual behaviour in male chicks. Journal of Endocrinology. 1978; 77: 149-150

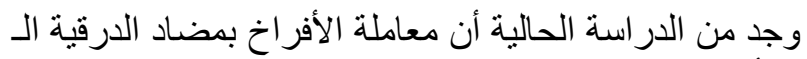
أدت إلى ارتفاع معنوي في الصفات الجنسية الذكرية

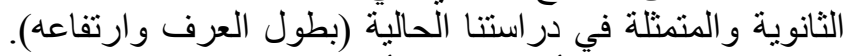

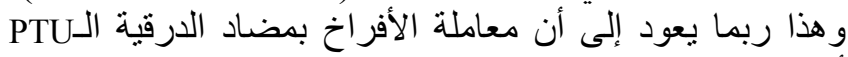
أدى إلى ارتفاع معنوي في تركيز هردي الى هون التستوستيرون

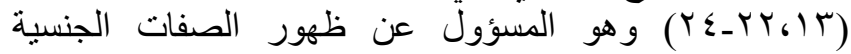

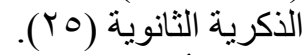

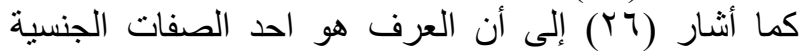
الذكرية الثانوية وان نمو العرف يتحفز بواسطة الاندروجينات،

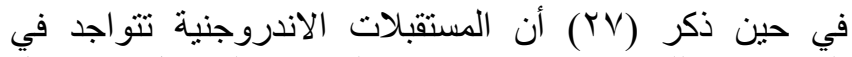

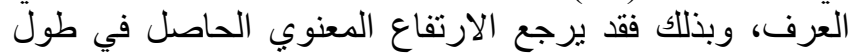
العرف وارتفاعه الذي وجد في دراستنا الحالية إلى الارتفاع

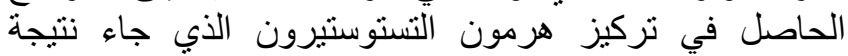
المعاملة بمضاد الدرقية الـ تركيز هون

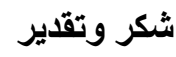

يشكر الباحثان عمادة كلية الطب البيطري، جامعة الموصل

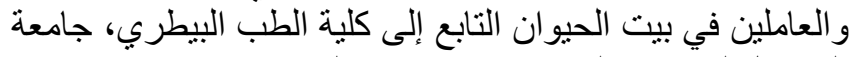
الموصل لدور هما الكبير في انجاز هذا البحث. البي.

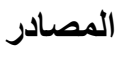

1. Cooke PS. Thyroid hormones development:a model for increasing testis growth and sperm production. Ann. NY Acad. Sci. 1991; 637: 122-132.

2. Jacquet MJ,; Seigneurin F, de Reviers M. Effect of thyroxine on testicular function, circulating luteinizing hormone and pituitary sensitivity to luteinizing hormone -releasing hormone in the cockerel.Brit.Poultry Sci. 1993; 34:803-814.

3. Dickson WM. Endocrinology, reproduction, and lactation.In:Swenson, MJ and Reece, WO (eds.), Duke's physiology of domestic animals ,London,Cornell University Press ;1996. p. 642644.

4. Mendis Handagama SMLC, Ariyaratne HBS. Differentiation of the adult Leydig cell population in the postnatal testis.Minireview.Biol.Reprod. 2001; 65:660-671.

5. Buzzard JJ. Morrison JR. o'Bryan MK;Song Q. Wreford NG. Developmental expression of thyroid hormone receptors in the rat testis. Biol. Reprod. 2000; 62:664-669.

6. Cooper D S. Antithyroid drugs. New Engl.J.Med. 1984; 311:13531362.

7. Yang Y. Gordon CJ. Regulated hypothermia in the hypothyroid rat induced by administration of propylthiouracil. Am. J. physiol. 1994 ; 272:1390-1395.

8. Levy M. Propylthiouracil hepatotoxicity. A review and case presentation. Clin.Pediatr. 1993; 32: 25-29. 